\title{
Detection of Radiation-Induced Apoptosis Using the Comet Assay
}

\author{
Seiichi WADA ${ }^{1,2)}$, Tran Van $\mathrm{KHOA}^{4)}$, Yasuhiko KOBAYASHI ${ }^{1)}$, Tomoo FUNAYAMA ${ }^{1)}$, Kazuo YAMAMOTO ${ }^{1,3)}$, \\ Masahiro NATSUHORI ${ }^{2}$ and Nobuhiko ITO $^{2)}$ \\ ${ }^{1)}$ Biotechnology Lab., JAERI-Takasaki, Watanuki-machi 1233, Takasaki, Gunma 370-1292, 2)Department of Veterinary Medicine, \\ Kitasato University, 35-1, Higashi 23 ban-cho Towada, Aomori 034-8628, ${ }^{3)}$ Department of Biomolecular Sciences, Graduate School of \\ Life Sciences, Tohoku University, Aramaki-Aoba, Aoba-ku, Sendai, Miyagi 980-8578, Japan and ${ }^{4)}$ Department of Biology and Medical \\ Geneties, Center of Radiation Health, 59-Ly, Kiet, Hanoi, Vietnam
}

(Received 8 November 2002/Accepted 22 July 2003)

ABSTRACT. The electrophoresis pattern of apoptotic cells detected by the comet assay has a characteristic small head and spread tail. Thi $\mathrm{s}$ image has been referred to as an apoptotic comet, but it has not been previously proven to be apoptotic cells by any direct method. In order to identify this image obtained by the comet assay as corresponding to an apoptotic cell, the frequency of appearance of apoptosis was examined using CHO-K1 and L5178Y cells which were exposed to gamma irradiation. As a method for detecting apoptosis, the terminal deoxynucleotidyl transferase mediated dUTP nick end labeling (TUNEL) assay was used. When the frequency of appearance of apoptotic cells following gamma irradiation was observed over a period of time, there was a significant increase in appearanc e of apoptosis when using the TUNEL assay. However, there was only a slight increase when using the comet assay. In order to verify th e low frequency of appearance of apoptosis when using the comet assay, we attempted to use the TUNEL assay to stain the apoptotic comets detected in the comet assay. The apoptotic comets were TUNEL positive and the normal comets were TUNEL negative. This indicate $\mathrm{s}$ that the apoptotic comets were formed from DNA fragments with 3'-hydroxy ends that are generated as cells undergo apoptosis. Th erefore, it was understood that the characteristic pattern of apoptotic comets detected by the comet assay corresponds to cells undergoing apoptosis.

KEY WORDS: apoptosis, comet assay, DNA fragment, gamma ray, TUNEL assay.

J. Vet.Med. Sci. 65(11): 1161-1166, 2003

Apoptosis is called programmed death and is controlled by the genetic mechanism [7]. Recent interest has focused not only on its role in tumor cell turnover and response to therapy, but also on its importance from a purely biological point.

Apoptosis leads to cytoskeletal disruption, cell shrinkage, membrane blebbing, and the disruption of deoxyribonucleic acid (DNA) into fragments, but does not lead to an inflammatory response in vivo [7]. One of the most characteristic biochemical phenomena of apoptosis is the fragmentation of DNA to 180-200 base-pairs (bp) by endonuclease acting in the linkage region between nucleosomes [22]. Because this DNA fragmentation produces a characteristic ladder pattern in agarose gel electrophoresis, this technique is used as an important standard indication of apoptosis. At present, enzymatic techniques are used for detecting DNA fragmentation at the individual cell level. The most common method is the terminal deoxynucleotidyl transferase mediated dUTP nick end labeling (TUNEL) assay [17,32]. When apoptosis occurs, working endonuclease changes DNA into single strand breaks (ssbs) or double strand breaks (dsbs) with 3'-hydroxy ends. Terminal deoxynucleotidyl transferase (TdT), used in the TUNEL assay, efficiently labels the 3'-hydroxy ends of DNA in cells undergoing apoptosis $[5,12]$. Also, in a similar method, termed in situ end labeling (ISEL) assay, 3'-hydroxy ends are labeled using DNA polymerase, or klenow [8]. These methods are widely used in the detection of DNA fragmentation in individual apoptotic cells.
The comet assay is a technique for detecting fragmented DNA at the level of the individual cell. It was originally developed for the detection of alkali-labile sites, ssbs and dsbs, which were induced either by genotoxic agents or by radiation, or for detecting the influence of environmental mutagens. The migration pattern of the nucleus observed in this method has the appearance of a comet, and the fluorescent intensity and length of its tail are correlated to the number of DNA strand breaks which have been induced by radiation or chemical substances. Therefore, this method is used for the evaluation of various genotoxic agents [13]. Furthermore, it is considered that this method could be used for detecting DNA fragmentation in an apoptotic cell [20]. As the frequency of occurrence of apoptosis increases, the tail moment and tail length, which are parameters correlated to the degree of DNA damage measured by the comet assay, both increase $[11,14]$. In practice, an image of a different type from the normal comet image is observed [24]. Therefore, the comet assay can be used to detect a cell undergoing apoptosis [6].

However, in many studies, the frequency of appearance of apoptotic cells using the comet and the other assay was compared $[4,9,16,31]$, the image of a different type from normal comet image is not directly proved to be a cell undergoing apoptosis.

Therefore, in this study, we compared the frequency of appearance of apoptotic cells using the comet and the TUNEL assay based on the detection of DNA fragmentation and conducted simultaneously the comet assay and 
immuno-chemical staining. Then, we attempted to directly identify the cells undergoing apoptosis, which form the characteristic image in the comet assay.

\section{MATERIALS AND METHODS}

Cell culture: CHO-K1 and L5178Y cells (RIKEN GENE BANK, Japan) were derived from Chinese hamster ovary and mouse leukemia, respectively. The CHO-K1 cells were grown in Ham's F12 medium (Sigma, St. Louis, U.S.A.) supplemented with $10 \%$ fetal bovine serum (FBS) (Nichirei, Tokyo, Japan) and $0.1 \mathrm{mg} / \mathrm{ml}$ kanamycin in $10 \mathrm{~cm}$ diameter culture dishes. L5178Y cells were grown in RPMI (Sigma) medium supplemented with $10 \% \mathrm{FBS}$ and $0.1 \mathrm{mg} / \mathrm{ml}$ kanamycin in $10 \mathrm{~cm}$ diameter culture dishes. Cells were incubated at $37^{\circ} \mathrm{C}$ in a humidified atmosphere of $5 \% \mathrm{CO}_{2}$ and 95\% air.

Irradiation: Cells were irradiated using a ${ }^{60} \mathrm{Co}$ source at a dose rate of $2.0 \mathrm{~Gy} / \mathrm{min}$. Dose was measured with thermoluminescent dosimeter. The cells were removed from the culture dishes using $0.05 \%$ trypsin and suspended at a density of $1 \times 10^{5} \mathrm{cell} / \mathrm{m} l$ with the medium in a tube. Cells suspended with the medium were irradiated at room temperature, and were re-incubated at $37^{\circ} \mathrm{C}$ immediately after irradiation.

Comet assay: Cells were re-suspended in phosphate-buffered saline (PBS, pH 7.4). Seventy-five $\mu l$ of $1 \%$ agarose GP-42 (Nacalai Tesque, Kyoto, Japan) was quickly layered on slide glasses and covered with another slide. The sandwiched slide glasses were placed on ice to allow the agarose to gel. The cell suspension was mixed 1:1 (volume/volume) with $2 \%$ agarose-LGT GP-42 (Nacalai Tesque), and $75 \mu \mathrm{l}$ of the cell mixture was quickly layered in the same manner after the removal of the covering slide glasses. Finally, 75 $\mu l$ of agarose GP-42 was again quickly layered on top. The slide glasses were placed immediately in a chilled nucleus lysing solution of $2.5 \mathrm{M}$ sodium chloride $(\mathrm{NaCl}), 100 \mathrm{mM}$ ethylenediamine tetraacetic acid tetrasodium salt ( $\mathrm{Na}_{4}$ EDTA), $1 \%$ sarkosyl, $10 \%$ dimethyl sulfocide (DMSO), and $1 \%$ Triton $\mathrm{X}-100$ and kept at $4{ }^{\circ} \mathrm{C}$ in the dark for $60 \mathrm{~min}$.

The slides were placed on a horizontal gel electrophoresis platform and covered with a chilled neutral solution consisting of $90 \mathrm{mM}$ Tris, $2 \mathrm{mM}$ ethylenediamine tetraacetic acid disodium salt ( $\mathrm{Na}_{2}$ EDTA) and $90 \mathrm{mM}$ boric acid $(\mathrm{pH} 8)$ for $1 \mathrm{hr}$. Electrophoresis was conducted at $4{ }^{\circ} \mathrm{C}$ in the dark for $30 \mathrm{~min}$ at $25 \mathrm{~V}(0.89 \mathrm{~V} / \mathrm{cm})$. The slide glasses were washed with $300 \mathrm{mM}$ sodium hydroxide $(\mathrm{NaOH})$ and $1 \mathrm{mM}$ $\mathrm{Na}_{2}$ EDTA and then rinsed gently with $400 \mathrm{mM}$ Tris $(\mathrm{pH}$ 7.5) to neutralize the excess alkali.

Each dried gel sample was stained with $20 \mu \mathrm{g} / \mathrm{m} l$ ethidium bromide and covered with a coverslip. Using a fluorescence microscope equipped with a green filter (Olympus, Tokyo), the frequency of apoptotic cells was detected by scoring apoptotic comets and non-apoptotic comets. About 500 cell were scored and experiments were repeated at least three times.
TUNEL assay: Cells were resuspended in PBS and were fixed for $15 \mathrm{~min}$ on ice with $1 \%$ buffered paraformaldehyde in PBS. Cells were dried on the slides and were stained with the ApopTag kit (Intergen, N.Y., U.S.A.). The slides were rehydrated in PBS and treated with TdT buffer ( $7 \mu l$ of TdT reaction buffer, $3 \mu l$ of TdT, $10 \mu l$ of equilibration buffer) for $60 \mathrm{~min}$ at $37^{\circ} \mathrm{C}$ in a humidified chamber. Peroxidaseconjugated anti-digoxigenin antibodies were added for 30 min at room temperature in a humidified chamber followed by washing with PBS buffer. The slides were stained by diaminobenzidine (DAB) solution and counterstained in $0.5 \%$ methyl green. The samples were examined by light microscopy. About 1,000 cells were scored and experiments were repeated at least three times.

Combined assay (Comet assay and TUNEL assay): Cells embedded in agarose gel were prepared as described above. Samples were treated with lysing solution and electrophoresis was performed. The samples were washed with alkaline solution and then rinsed gently with Tris buffer to neutralize the excess alkali. Air dried agarose gels were labeled with digoxigenin-dUTP using klenow (1 U/slide, Boehriger Mannheim, Tokyo) at $37^{\circ} \mathrm{C}$ for $60 \mathrm{~min}$, followed by washing with PBS. The agarose gels were stained with ApopTag kit (Intergen) for $60 \mathrm{~min}$ at $37^{\circ} \mathrm{C}$ in a humidified chamber. After washing the agarose gels with PBS, Fluorescein isothiocyanate (FITC)-conjugated anti-digoxigenin antibodies were added for $60 \mathrm{~min}$ at room temperature. The agarose gels were washed with PBS, stained with $0.5 \mu \mathrm{g} / \mathrm{m} l$ 4'-6diamidino-2-phenylindole (DAPI) and covered with a coverslip. Fluorescence images were observed using a fluorescence microscope (Olympus) and were collected with digital camera (Olympus).

\section{RESULTS}

DNA damage can be estimated in individual cells by the comet assay. Figure 1 is a photograph of a comet observed $24 \mathrm{hr}$ following irradiation with gamma rays (8 Gy). The photograph in Fig. 1 shows a typical image of a migrated cell nucleus with DNA strand breaks. The lower image in Fig. 1 shows that a small portion of the DNA remained at the origin, and that the greater portion of the DNA has fragmented and has migrated sufficiently to make the tail. This characteristic image is called a teardrop, pear-shaped, or large fan-like tail [3, 16, 20, 22, 34]. It is understood that this finely cut, spread, migrated DNA is indicative of an apoptotic cell. In this paper we refer to this image as an apoptotic comet.

The frequency of appearance of apoptotic comets and TUNEL positive cells was detected using the comet method and the TUNEL assay which is recognized method for detecting apoptotic cells. These were carried out using $\mathrm{CHO}-\mathrm{KI}$ cells at 24,48 , and $72 \mathrm{hr}$ following gamma irradiation $(8 \mathrm{~Gy}$ ). Figure 2 shows the frequency of appearance of apoptosis versus time when using both the comet method and the TUNEL assay following irradiation. When analyzing by the TUNEL assay, the frequency of appearance of 


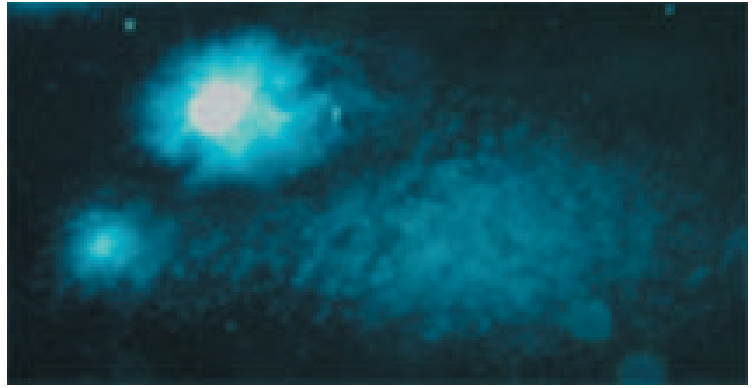

Fig. 1. Fluorescence photomicrograph of a comet of CHO-K1 at $24 \mathrm{hr}$ after exposure to $8 \mathrm{~Gy}$ gamma radiation. The comet images were stained with 4'-6-diamidino-2-phenylindole (DAPI) and were observed with a DAPI emission filter. The upper cell represents a normal comet image. The lower cell is considered to be an image of a typical comet undergoing apoptosis that has a small head and a spread tail. This image is called an apoptotic comet.

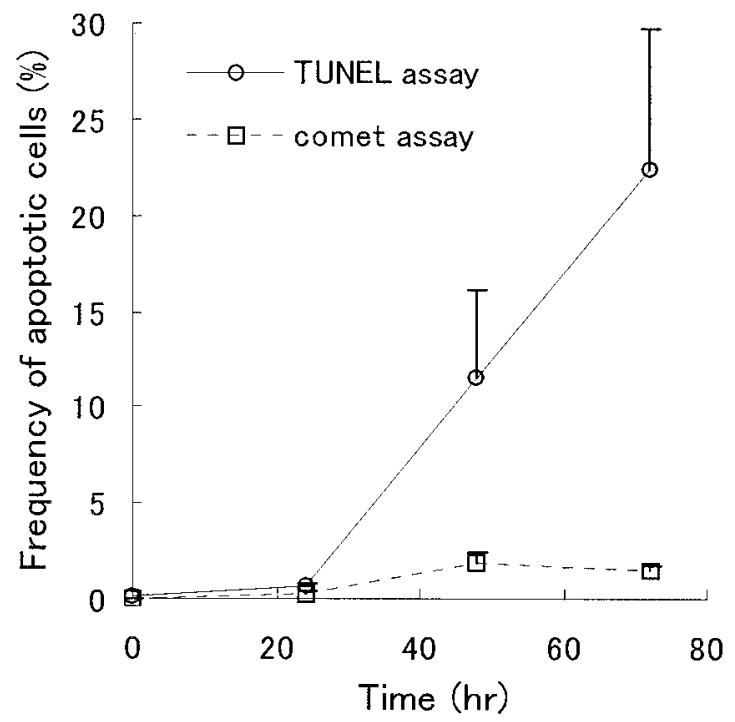

Fig. 2. The frequency of apoptotic cells detected by the comet assay $(\ldots . . . . . .$.$) and the terminal deoxynucleotidyl$ transferase mediated dUTP nick labeling (TUNEL) assay (- $\mathrm{O}-$ ) on $\mathrm{CHO}-\mathrm{K} 1$ after exposure to $8 \mathrm{~Gy}$ gamma radiation. The values presented are means \pm standard error (SE). At least three experiments were performed at each time interval. The frequencies of occurrence of apoptotic cells were significantly different at $48 \mathrm{hr}$ and $72 \mathrm{hr}$ after irradiation between assays.

apoptosis increased significantly over time. However, when analyzing by the comet assay, the frequency of appearance of apoptosis increased significantly less, by only approximately $1.5 \%$ at $72 \mathrm{hr}$ following irradiation. For both methods, the frequency of appearance of apoptotic cells was approximately the same at $24 \mathrm{hr}$ after irradiation. However, significant differences in the results were observed between the two methods at 48 and $72 \mathrm{hr}$ after irradiation. We next measured the frequency of appearance of apoptosis by both

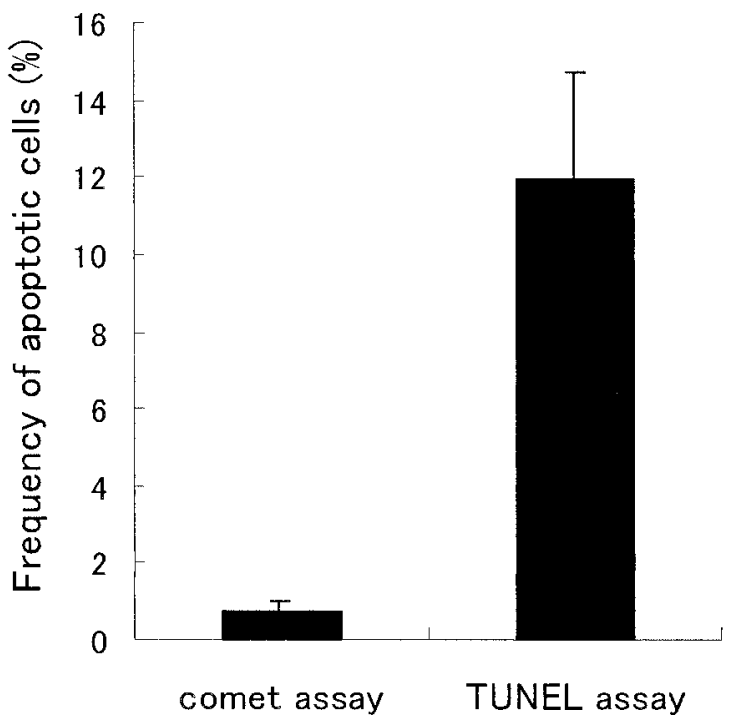

Fig. 3. The frequency of apoptotic cells detected by the TUNEL and the comet assays on the L5178Y at $48 \mathrm{hr}$ after exposure to $4 \mathrm{~Gy}$ gamma radiation. The values are means \pm SE. At least three experiments were performed. The percentage of apoptotic cells in the L5178Y cells were also different between both assays and less with the comet assay than with the TUNEL assay.

A

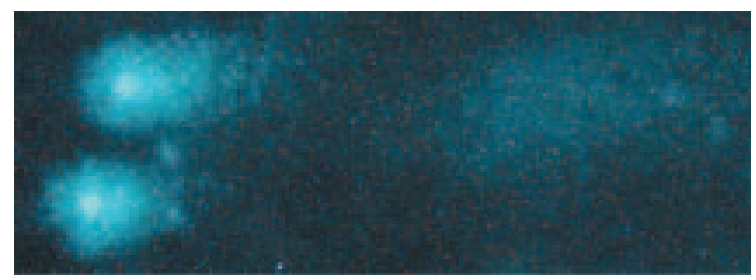

B

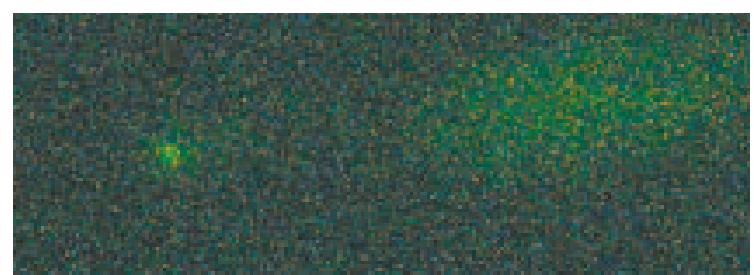

Fig. 4. Fluorescence photomicrograph of a comet of CHO-K1 at $72 \mathrm{hr}$ after exposure to $8 \mathrm{~Gy}$ gamma radiation. After lysis and electrophoresis, the $3^{\prime} \mathrm{OH}$ terminals were labeled with klenow and TdT. Panel A shows comet images observed with the DAPI emission filter. Normal comets and an apoptotic comet were observed. Panel B shows the same image as panel A observed with a fluorescein isothiocyanate (FITC) emission filter. The signal of the FITC was not observed on the normal comets. However, the signal of the FITC was observed on the apoptotic comet.

the TUNEL assay and the comet assay on L5178Y cells, which have previously been observed to display the typical ladder pattern of apoptotic cells, from $24 \mathrm{hr}$ to $96 \mathrm{hr}$ after irradiation [33]. Figure 3 presents the frequency of appear- 
ance of apoptosis at $48 \mathrm{hr}$ following gamma irradiation (4 Gy). The frequency of appearance of apoptosis was $10 \%$ when measured by the TUNEL assay, but was only $1 \%$, at most, when measured by the comet assay. With L5178Y, the frequency of appearance of apoptosis was considerably different. With the comet assay, the frequency of appearance of apoptosis was lower than observed with the TUNEL assay.

If the characteristic apoptotic comet detected by the comet assay corresponds to an apoptotic cell, then that comet should be TUNEL positive. So we experimented to see if the apoptotic comet would be directly stained by the TUNEL assay. The agarose gel was dried after electrophoresis, and was then stained with FITC using TdT and klenow. DAPI was used for Counterstaining. DAPI was stained blue, and FITC was stained green in this experiment. Figure 4A was observed using an emission filter for DAPI, and Fig. 4B was observed using an emission filter for FITC. When we observed the gel using the emission filter for DAPI, we observed both normal comets and apoptotic comet. On the other hand, when we observed the gel using the emission filter for FITC, the apoptotic comet was stained by FITC but the normal comets were not stained by FITC. When the comet assay and the TUNEL assay were combined, the apoptotic comets were stained by FITC. This result indicates that this method cannot detect all apoptotic cells. However, it was confirmed that the apoptotic comets detected in the comet assay corresponded to apoptotic cells.

\section{DISCUSSION}

The comet assay is available as a means of detecting DNA damage in individual cells. In apoptotic cells there are approximately 60,000 DNA strand breaks generated per cell [22]. With the comet assay, 100 DNA strand breaks per cell are generally sufficient for DNA damage to be detected [25]. For this reason, it is predicted that the many DNA fragments in an apoptotic cell would facilitate detection by the comet assay. Now the characteristic image in comet assay as described above is regard as an apoptotic cell.

However, this study has shown that the frequency of appearance of apoptosis by the comet assay when compared to the TUNEL assay did not match.

The DNA of cells undergoing apoptosis is initially cut to fragments sized approximately $50 \mathrm{kbp}$ to $300 \mathrm{kbp}$. These are then cut further into fragments of $200 \mathrm{bp}$ [10] and it is common to utilize the characteristic ladder pattern observed in agarose gel electrophoresis as a method for detecting DNA fragmentation. The comet assay can detect DNA fragments of size $50 \mathrm{kbp}$, but cannot detect the smaller fragments of approximately $200 \mathrm{bp}$ [30]. The tail will disappear since such small fragments of DNA diffuse in the agarose gel and exhibit greater migration during electrophoresis [21]. For this reason, the DNA fragments that contribute to the presentation of the ladder pattern cannot be observed by the comet assay. In other words, the apoptotic cell is undetectable once it has advanced to the later stages of apoptosis.
Furthermore, the translocation of phosphatidylserine to the cell membrane is one of the indicators of apoptosis in cells. Apoptotic cells can be detected using the binding of Annexin $\mathrm{V}$ to the exposed portion of phosphatidylserine [26]. An experimental system was developed which detects the translocation of phosphatidylserine, and simultaneously determines DNA damage by the comet assay [2]. It is understood that a cell is labeled by propidium iodide (PI) when the membrane becomes permeable after cell death. A cell that is positive for both PI and for Annexin V is a necrotic cell, while a cell that is negative for PI but positive for Annexin V is an apoptotic cell. When the comet assay was used on cells of the apoptosis fraction that were Annexin V positive and PI negative, apoptotic comets were not only observed but also condensed particles, called remnants, were also observed [2]. The remnants were the residue remaining after diffusion of most of the DNA by cytolysis or electrophoresis, leaving part of the nuclear matrix. Consequently, in the comet assay, the cells presenting as apoptotic comets do not represent all of the apoptotic cells, so this method underestimates the frequency of appearance of apoptotic cells.

Recently, a diffusion assay, which is a method designed to prevent migration of DNA fragments by omitting electrophoresis, was developed. In this method, DNA fragments diffused inside an agarose gel during cytolysis and the apoptotic cells presented a halo pattern that is different from that of non-apoptotic cells. The frequency of appearance of apoptotic cells by morphological assay was similar to the frequency of appearance of cells presenting with the halo, over a period of time [28, 29].

Furthermore, flow cytometry is also frequently used as a technique for detecting apoptotic cells [23]. Apoptotic cells stained with DNA-binding dyes decrease in fluorescent intensity as the DNA was fragmented. When the frequency of apoptosis over time was compared using flow cytometry and the comet assay, the apoptotic comets appeared at an earlier stage than the detection of apoptosis by flow cytometry $[10,23]$. Moreover, the translocation of phosphatidylserine is an early reaction in advanced apoptosis. The frequency of appearance of apoptotic comets, and Annexin $\mathrm{V}$ reactivity, were shown to increase correspondingly [9], or DNA fragmentation precedes Annexin V reactivity [1]. Consequently, the apoptotic comet observed in the comet assay appears at an early stage of apoptosis.

Therefore, this study has shown that the frequency of appearance of TUNEL-positive cells and apoptotic comet cells did not match after 48 and $72 \mathrm{hr}$ following irradiation and that the apoptotic comet occurred at a lower frequency than TUNEL-positive cells.

Recently, there have been reports of immuno-chemical staining directly onto the comet image, and hybridization techniques have been conducted on the comet image using suitable probes, including probes from all genomes [18,27]. By combining the comet assay and fluorescence in situ hybridization (FISH), structural analysis of the nucleus became possible, as well as examination of the break and 
repair of regions of specific sequences in individual cells. Also, there has been success to directly label the site of the DNA strand breaks induced by hydrogen peroxide $\left(\mathrm{H}_{2} \mathrm{O}_{2}\right)$ in the comet image using the TdT [15].

We labeled apoptotic DNA fragments on the comet image using the TdT to conduct direct immuno-chemical staining. In this experiment, insufficient signals were observed in the comet images from the TdT alone. By pretreatment with klenow, signals from FITC were observed in the images appearing as apoptotic comets, and signals from FITC were not observed in the images appearing as normal comets. This study proved directly that the apoptotic comets contain DNA fragments with 3'-hydroxy ends, which are generated as cells undergo apoptosis, further demonstrating that the image of the apoptotic comet corresponds to apoptotic cells.

With an apoptotic comet, DNA fragments diffuse within the agarose gel and migrate a significant distance under electrophoresis [24]. For this reason, an apoptotic comet exhibits a lower fluorescent intensity than a cell with nonapoptotic DNA damage, even if the apoptotic comet has been stained with DNA-binding dyes [19]. This would indicate that because the superstructure of DNA dose not completely unwind in a cell with non-apoptotic DNA damage, such as DNA damage induced by $\mathrm{H}_{2} \mathrm{O}_{2}$, TUNEL staining of comet appear as bright clump [15]. To the contrary, the superstructure of DNA almost unwinds and various DNA fragments contain in apoptotic cells. Thus it was considered that FITC signal was observed throughout apoptotic comet image.

Although both the comet assay and the TUNEL assay detect DNA strand breaks, it is considered that the comet assay has greater specificity at the molecular level. Nonspecific DNA strand breaks which the enzyme of the TUNEL assay can not label, can be detected during the unwinding of chromatin and migration under electrophoresis using the comet assay [9]. It was understood that the signal of FITC could not be fully detected by using the TdT alone, even though the TdT with a high efficiency label was used [12].

However, the FITC signal in each apoptotic comet could be observed by conducting pretreatment with klenow. This combined technique labeled not only the 3' overhang ends and the blunt 3'-hydroxy ends of the DNA breaks in apoptotic cells, but also the 5' overhang ends which were labeled using klenow. Furthermore, when the 5' overhang end is labeled with nucleotide by klenow, it is changed to a blunt end. It is understood that this blunt end is labeled more efficiently by TdT. In other words, the FITC signal increases as many nucleotides in the apoptotic comet are labeled.

When apoptosis occurs in tumor tissue in vivo, because the cells in later stages of apoptosis are removed from the tumor tissue by phagocytosis, it is nearly impossible to detect apoptotic cells. So it is important to be able to detect the early stages of apoptosis in vivo. Though it is difficult to distinguish apoptotic cells and non-apoptotic cells with morphological assay, it is easy to distinguish with the comet assay. This indicates that not only can DNA damage and repair be measured, but also early apoptosis in vivo can be observed with good specificity with the comet assay.

In conclusion, not all apoptotic cells are detectable by the comet assay. However, we have shown that the apoptotic comets observed by the comet assay are apoptotic cells.

ACKNOWLEDGEMENTS. The authors thank Dr. S. Ueno of Department of Veterinary Medicine, Kitasato University, for valuable suggestions.

\section{REFERENCES}

1. Bacsó, Z. and Eliason, J. E. 2001. Measurement of DNA damage associated with apoptosis by laser scanning cytometry. Ctyometry 45: 180-186.

2. Bacsó, Z., Everson, R. B. and Eliason, J. F. 2000. The DNA of Annexin V-binding apoptotic cell is highly fragmented. Cancer Res. 60: 4623-4628.

3. Bock, C., Dube, A., Greulich, K. O. and Gupta, P. K. 1999. Identification by microscopically controlled comet assay of peritoneal macrophages in a mixture of peritoneal exudate for DNA strand break analysis. Mutat. Res. 439: 171-181.

4. Cregan, S. P., Smith, B. P., Brown, D. L. and Mitchel, R. E. J. 1999. Two pathways for the induction of apoptosis in human lymphocytes. Int. J. Radiat. Biol. 75: 1069-1086.

5. Cregan, S. P., Boreham, D. R., Walker, P. R., Brown, D. L. and Michel, R. E. J. 1994. Modification of radiation-induced apoptosis in radiation-or hyperthemia-adapted human lymphocytes. Biochem. Cell Biol. 72: 475-482.

6. Dikomey, E., Dahm-daphi, J., Brammer, I., Martensen, R. and Kaina, B. 1998. Correlation between cellular radiosensitivity and non-repaired double-strand breaks studied in nine mammalian cell lines. Int. J. Radiat. Biol. 73: 269-278.

7. Fairbairn, D. W., Walburger, D. K., Fairbairn, J. J. and O'Neill, K. L. 1996. Key morphologic changes and DNA strand breaks in human lymphoid cells: Discriminating apoptosis from necrosis. Scanning 18: 407-416.

8. Fujita, K., Kawarada, Y., Terada, K., Sugiyama, T., Ohyama, H. and Yamada, T. 2000. Quantitative detection of apoptotic thymocytes in low-dose $\mathrm{X}$-irradiated mice by an anti-singlestranded DNA antibody. J. Radiat. Res. 41: 139-149.

9. Godard, T., Deslandes, E., Lebailly, P., Vigreux, C., Sichel, F., Poul, J. M. and Ganuduchon, P. 1999. Early detection of stauroaporone-induced apoptosis by comet assay and Annexin V assays. Histochem. Cell Biol. 112: 155-161.

10. Godard, T., Deslandes, E., Lebailly, P., Vigreux, C., Poullain, L., Sichel, F., Poul, J. M. and Gauduchon, P. 1999. Comet assay and DNA flow cytometry analysis of staurosporineinduced apoptosis. Cytometry 36: 117-122.

11. Gopalakrishna, P. and Khar, A. 1995. Comet assay to measure DNA damage in apoptotic cells. J. Biochem. Biophys. Methods 30: $69-73$.

12. Gorczyca, W., Gong, J. and Darzynkiewicz, Z.1993. Detection of DNA strand breaks in individual apoptotic cells by the in situ terminal deoxynucleotidyl transferase and nick translation assays. Cancer Res. 53: 1945-1951.

13. Henderson, L., Wolfreys, A., Fedyk, J., Bourner, C. and Windebank, S. 1998. The ability of the comet assay to discriminate between genotoxins and cytotoxins. Mutagenesis 13: 89-94.

14. Humar, B., Muller, H. and Scott, R. J. 1997. Elevated frequency of p53-independent apoptosis after irradiation increases 
levels of DNA breaks in ataxia telangiectasia lymphoblasts. Int. J. Radiat. Biol. 72: 257-269.

15. Kindzelskii, A. L. and Petty, H. R. 1999. Ultrasensitive detection of hydrogen peroxide-mediated DNA damage after alkaline single cell gel electrophoresis using occultation microscopy and TUNEL labeling. Mutat. Res. 426: 11-22.

16. Kizilian, N., Wilkins, R. C., Reinhardt, P., Ferrarotto, C., Mclean, J. R. N. and Mcnamee, J. P. 1999. Silver-stained comet assay for detection of apoptosis. Biotechniques 27: 926930.

17. Kockx, M. M., Muhring, J., Knaapen, M. W. M. and de Meyer, G. R.Y. 1998. RNA synthesis and splicing interferes with DNA in situ end labeling techniques used to detect apoptosis. Am. J. Pathol. 152: 885-888.

18. McKelvey-Martin, V. J., Ho, E. T. S., McKeown, S. R., Johnston, S. R., McCarthy, P. J., Rajab, N. E. and Downes, C. S. 1998. Emerging applications of the single cell gel electrophoresis (comet) assay. I. Management of invasive transitional cell human bladder carcinoma. II. Fluorescent in situ hybridization comets for identification of damaged and repaired DNA sequence in individual cells. Mutagenesis 13: 1-8.

19. O'Callaghan, Y. C., Woods, J. A. and O'Brien, N. M. 2001. Limitations of the single-cell gel electrophoresis assay to monitor apoptosis in U937 and HepG2 cells exposed to $7 \beta$-hydroxycholesterol. Biochem. Phamacol. 61: 1217-1226.

20. Ohyama, H., Tamamoto, H. and Yanada, T. 1998. "Teardrop assay," a rapid and sensitive method for characterization of radiation-induced apoptotic DNA fragmentation in individual thymocytes. pp. 185-198. In: Apoptosis: Its Role and Mechanism. Business Center for Academic Societies.

21. Olive, P. L. 1999. Review. DNA damage and repair in individual cells: applications of the comet assay in radiobiology. Int. J. Radiat. Biol. 75: 395-405.

22. Olive, P. L. and Banath, J. P. 1995. Sizing highly fragmented DNA in individual apoptotic cells using the comet assay and a DNA crosslinking agent. Exp. Cell Res. 221: 19-26.

23. Olive, P. L., Frazer, G. and Banáth, J. P. 1993. Radiationinduced apoptosis measured in TK6 human B lymphoblast cells using the comet assay. Radiat. Res. 36: 130-136.
24. Olive, P. L., Johnston, P. J., Banath, J. P. and Durand, R. E. 1998. The comet assay: A new method to examine heterogeneity associated with solid tumors. Nat. Med. 4: 103-105.

25. Robert, S. M., Chen, B., Eric, W. A. and Joseph, L. R. 1998. Detection of DNA damage by the alkaline comet assay after exposure to low-dose gamma radiation. Radiat. Res. 149: 396400.

26. Roser, S., Pool-Zobel, B. L. and Rechkemmer, G. 2001. Contribution of apoptosis to responses in the comet assay. Mutat. Res. 497: 169-175.

27. Santos, S. J., Singh, N. P. and Natarajan, A. T. 1997. Fluorescence in situ hybridization with comets. Exp. Cell Res. 232: 407-411.

28. Singh, N. P. 2000. A simple method for accurate estimation of apoptotic cells. Exp. Cell Res. 256: 328-337.

29. Singh, N. P. 2000. Microgel for estimation of DNA strand breaks, DNA protein crosslink and apoptosis. Mutat. Res. 455: 111-127.

30. Singh, N. P., Stephens, R. E., Singh, H. and Lai, H. 1999. Visual quantification of DNA double-strand breaks in bacteria. Mutat. Res. 429: 159-168.

31. Siles, E., Villalobos, M., Jones, L., Guerrero, R., Eady, J. J., Valenzuela, M. T., Núnez, M. I., Mcmillan, T. J. and Ruiz de Almodo, J. M. 1998. Apoptosis after gamma irradiation, Is it an important cell death modality? Br. J. Cancer 78: 1594 1599.

32. Tateyama, H., Tada, T., Hattori, H., Murase, T., Li, W. X. and Eimoto, T. 1998. Effects of prefixation and fixation times on apoptosis detection by in situ end-labeling of fragmented DNA. Arch. Pathol. Lab. Med. 122: 252-255.

33. Tauchi, H. and Sawada, S. 1994. Analysis of mitotic cell death caused by radiation in mouse leukaemia L5178Y cells: apoptosis is the ultimate form of cell death following mitotic failure. Int. J. Radiat. Biol. 65: 449-455.

34. Wilkins, R. C., Wilkinson, D., Maharaj, H. P., Bellier, P. V., Cybulski, M. B. and McLean, J. R. N. 2002. Differential apoptotic response to ionizing radiation in subpopulations human white blood cells. Mutat. Res. 513: 27-36. 